\title{
Diagnosis and treatment challenges of parathyroid carcinoma in a 27-year-old woman with multiple lung metastases
}

\author{
Liudmila Rozhinskaya', Ekaterina Pigarova', Ekaterina Sabanova', Elizaveta Mamedova', Iya Voronkova², \\ Julia Krupinova4, Larisa Dzeranova4, Anatoly Tiulpakov3, Vera Gorbunova5, Nadezhda Orel5, \\ Artur Zalian5, Galina Melnichenko ${ }^{4}$ and Ivan Dedov ${ }^{4}$
}

Departments of ${ }^{1}$ Neuroendocrinology and Bone Diseases, ${ }^{2}$ Pathomorphology, ${ }^{3}$ Department and Laboratory of Inherited Endocrine Disorders, ${ }^{4}$ Endocrinology Research Centre, Moscow, Russia, and ${ }^{5}$ Department of Chemotherapy, Cancer Research Center, Moscow, Russia

Correspondence should be addressed to J Krupinova Email

j.krupinova@gmail.com

\section{Summary}

Parathyroid carcinoma is an extremely rare disorder with little treatment options. It could be misdiagnosed in medical centers with little experience in management of such cases. Our clinical case shows that the initial misdiagnosis of a parathyroid carcinoma in a young woman has led to the development of multiple lung metastases, thus making its treatment hardly possible. Initiation of treatment with sorafenib - a multi-kinase inhibitor approved for treatment of different types of cancer but not parathyroid carcinoma - has led to a significant decrease in the size of lung metastases and has prevented the progression of hyperparathyroidism, which is usually severe in cases of parathyroid carcinoma. The detection of a germline $C D C 73$ mutation in this patient has raised additional concerns about the necessity of periodic screening for early detection of renal, jaw and uterine lesions.

\section{Learning points:}

- Diagnosis of parathyroid carcinoma may be challenging due to the absence of reliable diagnostic criteria. Thus, thorough histological examination is needed using immunohistochemical staining of resected tissue in suspicious cases.

- $C D C 73$ genetic testing should be considered in patients with parathyroid carcinoma.

- Sorafenib may be a promising treatment of patients with parathyroid carcinoma with distant metastases.

\section{Background}

Parathyroid carcinoma (PC) is a rare neoplasm with a prevalence of only $0.005 \%$ of all malignancies, and less than $1 \%$ among patients with primary hyperparathyroidism $(1,2,3)$. The diagnosis can be challenging due to the rarity of the disease, similarity of clinical features to parathyroid adenoma and lack of reliable morphological signs of the tumor. This may lead to a delayed diagnosis and poor prognosis, with PC having already spread. PC can be the first manifestation of hyperparathyroidism-jaw tumor syndrome caused by germline mutations in $C D C 73$ tumor suppressor gene (4).

Treatment options for patients with metastatic PC are limited especially if complete tumor removal cannot be performed. Chemotherapy and radiotherapy failed to show any significant effect on the course of the disease $(5,6)$.

We report the case of a 27-year-old woman with PC previously misdiagnosed as a benign thyroid nodule and multiple lung metastases, who was referred to our 
institution with suspected primary hyperparathyroidism. After thorough examination, the diagnosis of hyperparathyroidism-jaw tumor syndrome was established based on genetic testing, and successful treatment of PC lung metastases with a multi-kinase inhibitor sorafenib was initiated.

\section{Case presentation}

A 27-year-old woman was referred to our tertiary care center with muscle weakness, polyuria, polydipsia, appetite loss and morning nausea. About a year before this admission, a lung tumor was visualized on frontal chest X-ray during prophylactic medical examination. Further, a computed tomography (CT) scan revealed $2.9 \times 2.8 \mathrm{~cm}$ and $2.2 \times 2.0 \mathrm{~cm}$ diameter round tumors in the lower lobe of the right lung (S9, S10), as well as smaller masses, $0.6 \mathrm{~cm}$ and $0.8 \mathrm{~cm}$, in the $\mathrm{S} 2$ and the $\mathrm{S} 8$ respectively (Fig. 1).

\section{Investigation}

Transthoracic puncture of the largest tumor followed by immunocytochemical analysis showed an absence of expression of primary lung adenocarcinoma markers, such as thyroid transcription factor-1 (TTF-1), cytokeratin 7 (CK7) and cytokeratin 20 (CK20) in tumor tissue, but positive expression of cytokeratin 19 (CK19).

CT scan image was also notable for multiple bone lytic changes in the cervical and thoracic vertebrae, ribs, sternum and scapulae. On radionuclide bone imaging, osteitis fibrosa cystica was suspected and consequent evaluation revealed an elevation of PTH up to $249 \mathrm{pmol} / \mathrm{L}$ (reference range 1.6-6.9) and serum calcium level up to $4.19 \mathrm{mmol} / \mathrm{L}$ (2.10-2.55). There were no ultrasonographic signs of parathyroid neoplasms, and 99mTc-sestamibi scintigraphy failed to localize any focal areas of increased radiotracer uptake.

It is noteworthy that eight years before this admission, the patient underwent hemithyroidectomy for a $3.1 \mathrm{~cm}$ diameter tumor diagnosed as a thyroid nodule.
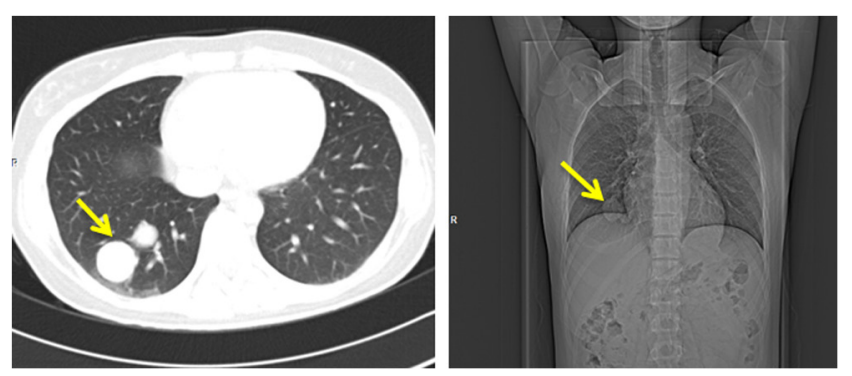

Figure 1

Lung tumors (indicated by arrows) on CT scan.
The resected tissue was histologically described at that hospital as a cyst with hemorrhage and inflammation in the capsule. There was no available information about serum calcium and PTH levels at that time. Considering clinical data consistent with manifest primary hyperparathyroidism with severe hypercalcemia, signs of osteitis fibrosa cystica, myopathy without visualized source of PTH overproduction and inability to exclude ectopic PTH production by the lung tumors, the surgical removal of the neoplasms was recommended.

\section{Treatment}

To correct hypercalcemia, the patient received $4 \mathrm{mg}$ of zoledronic acid intravenously, which lowered total serum calcium level to $2.8 \mathrm{mmol} / \mathrm{L}$.

The patient underwent thoracoscopic lower lobe resection of the right lung with two $2.9 \times 2.8 \mathrm{~cm}$ and $2.2 \times 2.0 \mathrm{~cm}$ diameter tumors were removed. The smaller ones found in S2 and S8 were not available for surgical excision. PTH and total serum calcium levels decreased to $15 \mathrm{pmol} / \mathrm{L}$ and $1.9 \mathrm{mmol} / \mathrm{L}$ respectively. After administering calcium supplements (calcium carbonate $2500 \mathrm{mg}$ twice a day) and active form of vitamin D3 (alfacalcidol $2 \mu \mathrm{g}$ a day), serum calcium level normalized and PTH level decreased to $8 \mathrm{pmol} / \mathrm{L}$.

We performed histological and immunohistochemistry analysis of lung neoplasms and tissues after hemithyroidectomy.

The presumed thyroid nodule specimen proved to be a parathyroid gland neoplasm composed of basophil and chromophobe cells, which intensively expressed PTH and was surrounded by a thick fibrous capsule with the presence of intravascular emboli. Mean proliferative activity and receptor status were not assessed because of the small amount of the material. Findings were considered to be related to PC.

Material derived from the lung surgery was presented as a solid trabecular tumor consisting of basophil and chromophobe cells. Both cell types intensively expressed PTH, and moderately expressed chromogranin A and synaptophysin (Fig. 2A and B). The expression of adrenocorticotropic hormone, growth hormone, prolactin, calcitonin and TTF-1 was absent. The number of mitotic figures in 10 representative high power fields was 4, after reaction with phosphohistone H3 (pHH3) - 13 per 10 representative high power fields. Mean proliferative activity assessed by Ki-67 was 19\%.

Thus, clinical presentation and pathology data served the basis for diagnosing PC with multiple lung metastases. 


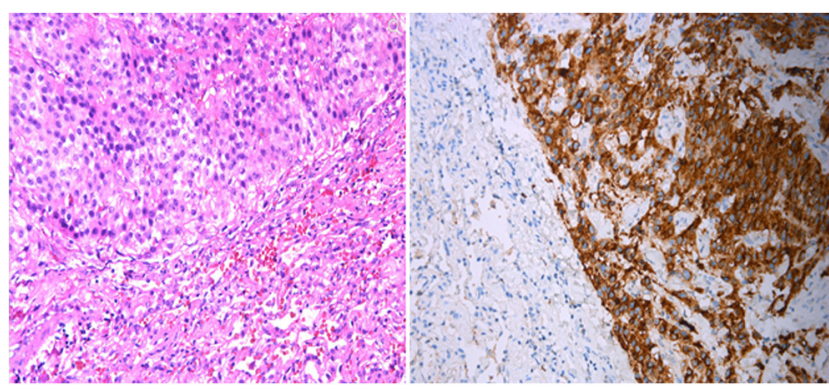

\section{Figure 2}

(A) Hematoxylin-eosin $(\times 400)$. Lung parenchyma with metastasis from the primary parathyroid carcinoma. (B) Immunohistochemical analysis of PTH expression ( $\times 400)$. Diffuse intensive expression of PTH by the lung metastasis from the primary parathyroid carcinoma.

Given the high prevalence of cell cycle division 73 gene (CDC73; NM_024529) mutations in patients with PC (up to $30 \%$ ), we performed next-generation sequencing (NGS) with a custom-designed Ion AmpliSeq gene panel, which included $C D C 73$, using a semiconductor sequencer Ion Torrent PGM (Thermo Fisher Scientific-Life Technologies). NGS revealed a novel nonsense mutation in $C D C 73$ c.496C > T (p.Q166X), leading to premature termination of translation and thus truncation of encoded parafibromin. The identification of a germline $C D C 73$ mutation enabled to diagnose hyperparathyroidism-jaw tumor syndrome; yet, no other features of the syndrome were present in our patient.

\section{Outcome and follow-up}

Five months after lung surgery, PTH was $7.2 \mathrm{pmol} / \mathrm{L}$ with high total serum calcium $(2.74 \mathrm{mmol} / \mathrm{L})$ and low 24 -h urinary excretion of calcium $(0.85 \mathrm{mmol} / 24 \mathrm{~h})$, which lead to a decrease of alfacalcidol and calcium. Diffusional MRI (DW-MRI) showed multiple metastatic lesions in lungs $0.2-0.5 \mathrm{~cm}$ in diameter.

Based on the reports of successful use of sorafenib for the treatment of metastatic PC by Dr Lerario et al., we started a targeted therapy with $400 \mathrm{mg}$ of sorafenib 2 times a day and $1250 \mathrm{mg}$ of calcium carbonate twice a day as well as $1 \mu \mathrm{g}$ of alfacalcidol daily $(7,8)$. The results of the laboratory examination 7 months after the initiation of sorafenib therapy were as follows: PTH $10.5 \mathrm{pmol} / \mathrm{L}$, total serum calcium $-2.42 \mathrm{mmol} / \mathrm{L}, 24$-h urine calcium $6.91 \mathrm{mmol} / 24 \mathrm{~h}$. DW-MRI made 7 months after the first examination did not visualize any of previously detected multiple focal masses. Given the increased ability to capture the fluorodeoxyglucose in the lungs according to the PET/CT scan and the absence of PTH normalization, sorafenib therapy was continued. A palmar-plantar erythrodysesthesia syndrome developed in a patient after initiation of sorafenib therapy so she locally applied a specialized antioxidant cream that helped to treat the lesions.

The patient is regularly observed in our center for the assessment of continuous treatment with sorafenib. Her palmar-plantar erythrodysesthesia has resolved. The overall follow-up period is now 22 months.

\section{Discussion}

The diagnosis of $\mathrm{PC}$ is challenging due to the lack of reliable diagnostic criteria. PC may be diagnosed years later when the patient exhibits metastases as in the case of our patient. The treatment of choice in PC is an en bloc resection of the mass with total ipsilateral thyroid loboistmectomy and central compartment lymphadenectomy (5), whereas in our patient, only parathyroidectomy with hemithyroidectomy was initially performed.

Treatment options for inoperable patients are very limited. Chemotherapy and radiotherapy failed to show any significant effect on the course of the disease. Recent reports have demonstrated radiofrequency ablation to be a promising option for these patients (6).

Given the strong evidence for the link between the inactivation of $C D C 73$ gene and malignant parathyroid tumor, CDC73 mutation screening and detection of parafibromin immunoreactivity have been suggested as diagnostic instruments of PC.

Increased angiogenesis in PC is a logical target for sorafenib that blocks angiogenesis (VEGFR-l, VEGFR-2, VEGFR-3, PDGFR- $\beta$, BRAF), cell proliferation, cell survival, all of which can be driven by the Raf-MAPK/ERK kinase-ERK pathway (9). Moreover, sorafenib effectively inhibits the activity of vascular endothelial growth factor receptors, platelet-derived growth factor receptor $\beta$, FLT-3, and c-Kit (10). Kinase inhibitors (KIs) have emerged as attractive therapeutic options for endocrine tumors. A recent study has shown that parathyroid tumors have high expression of VEGFR and PDGFR, providing a rationale for considering KIs for refractory PC $(7,8)$. Eventually, sorafenib seems to manage PC due to its ability to block angiogenesis. However, the precise anti-proliferative mechanisms of sorafenib in PC remain unclear.

\section{Declaration of interest}

The authors declare that there is no conflict of interest that could be perceived as prejudicing the impartiality of the research reported. 


\section{Funding}

Some parts of the research were done using funds of the grant of the President of the Russian Federation №MK-8689.2016.7 and some parts of the research were done using funds of the state assingnment.

\section{Patient consent}

Written informed consent has been obtained from the patient for publication of the article and accompanying images.

\section{Author contribution statement}

Liudmila Rozhinskaya supervised all diagnostic and treatment decisions, and contributed to the editing of the article. Pigarova Ekaterina was the attending physician of the patient and contributed to the writing of the article. Ekaterina Sabanova was the resident physician of the patient. Elizaveta Mamedova performed genetic testing and wrote the article. lya Voronkova performed morphological and immunohistochemical investigations. Julia Krupinova was the resident physician of the patient and contributed to the writing of the article. Larisa Dzeranova contributed to the editing of the article. Anatoly Tiulpakov interpreted the genetic results. Vera Gorbunova initiated and still monitors the treatment with sorafenib; also contributed to the editing of the article. Orel Nadezhda monitors the treatment with sorafenib; contributed to the editing of the article. Artur Zalianwas the patient's surgeon; Galina Melnichenko contributed to the writing and editing the article. Ivan Dedov supervised the writing and editing of the final article.

\section{References}

1 Hundahl SA, Fleming ID, Fremgen AM \& Menck HR 1999 Two hundred eighty-six cases of parathyroid carcinoma treated in the U.S. between 1985 and 1995: a National Cancer Data Base Report. The American College of Surgeons Commission on Cancer and the American Cancer Society. Cancer 86 538-544.
(doi:10.1002/(SICI)1097-0142(19990801)86:3<538::AIDCNCR25>3.0.CO;2-K)

2 Schantz A \& Castleman B 1973 Parathyroid carcinoma. A study of 70 cases. Cancer 31 600-605. (doi:10.1002/10970142(197303)31:3<600::AID-CNCR2820310316>3.0.CO;2-0)

3 Ippolito G, Palazzo FF, Sebag F, De Micco C \& Henry JF 2007 Intraoperative diagnosis and treatment of parathyroid cancer and atypical parathyroid adenoma. British Journal of Surgery 94 566-570. (doi:10.1002/bjs.5570)

4 Cetani F, Pardi E, Borsari S, Viacava P, Dipollina G, Cianferotti L, Ambrogini E, Gazzerro E, Colussi G, Berti P, et al. 2004 Genetic analyses of the HRPT2 gene in primary hyperparathyroidism: germline and somatic mutations in familial and sporadic parathyroid tumors. Journal of Clinical Endocrinology and Metabolism 89 5583-5591. (doi:10.1210/jc.2004-0294)

5 Shane E 2001 Parathyroid carcinoma. Journal of Clinical Endocrinology and Metabolism 86 485-493. (doi:10.1210/jcem.86.2.7207)

6 DasGupta R, Shetty S, Keshava NS, Gupta M, Paul MJ \& Thomas N 2014 Metastatic parathyroid carcinoma treated with radiofrequency ablation: a novel therapeutic modality. Australasian Medical Journal 7 372-375. (doi:10.4066/AMJ.2014.2084)

7 Lerario AM, Martin RM, Hoff AO, Nakaguma M, Castro G, Teixeira CH, Menezes M, Dalalio V \& Mendonca BB 2014 Sorafenib treatment improves refractory hypercalcemia in a patient with metastatic parathyroid carcinoma: a case report. In Endocrine Society's 96th Annual Meeting and Expo, June 21-24, Chicago (Abstract SUN0280). (Unpublished observations)

8 Busaidy NL, Cabanillas ME, Dadu R, Jimenez C, Habra MA, Vaporciyan A, Morris L, Perrier ND \& Hoff AO 2014 Metastatic parathyroid carcinoma and hypercalcemia responds to treatment with sorafenib. In Endocrine Society's 96th Annual Meeting and Expo, June 21-24, Chicago (Abstract SUN-0279). (Unpublished observations)

9 Gray-Schopfer V, Wellbrock C \& Marais R 2007 Melanoma biology and new targeted therapy. Nature 445 851-857. (doi:10.1038/ nature05661)

10 Stein MN \& Flaherty KT 2007 CCR drug updates: sorafenib and sunitinib in renal cell carcinoma. Clinical Cancer Research 13 3765-3770. (doi:10.1158/1078-0432.CCR-06-2844) 